\title{
The ignorance behind inconsistency toleration
}

\author{
María del Rosario Martínez-Ordaz*
}

\begin{abstract}
Inconsistency toleration is the phenomenon of working with inconsistent information without threatening one's rationality. Here I address the role that ignorance plays for the tolerance of contradictions in the empirical sciences. In particular, I contend that there are two types of ignorance that, when present, can make epistemic agents to be rationally inclined to tolerate a contradiction. The first is factual ignorance, understood as temporary undecidability of the truth values of the conflicting propositions. The second is what I call "ignorance of theoretical structure", which is lack of knowledge of relevant inference patterns within a specific theory. I argue that these two types of ignorance can be explanatory of the scientists' rational disposition to be tolerant towards contradictions, and I illustrate this with a case study from neutrino physics.
\end{abstract}

Keywords - Inconsistency toleration, factual ignorance, ignorance of theoretical structure, measurement of solar neutrinos' flux.

\section{Introduction}

A contradiction is a pair of propositions where one is a negation of the other. A set of propositions is trivial if every proposition is derivable from it. According to the Principle of Explosion, any set of propositions, if closed under classical logic or any other explosive logic, is trivial if containing a contradiction. Given the role that classical logic has played in the development and conceptual foundations of science and philosophy through time, it is not surprising that scientists and philosophers have regarded contradictions as extremely malignant.

There is a recurring view in the traditional literature of logic and philosophy of science which holds that the use of inconsistent information in the sciences entails the irrationality of the scientists. If assuming the basic principles of classical logic (or any other explosive logic), "an inconsistent theory implies any conceivable observational prediction as well as its negation and thus tells us nothing about the world" (Hempel, 2000: 79). Therefore, any scientist who consciously trusts such a theory must be irrational.

Pace these traditional intuitions, in recent decades, philosophers and historians of science have noticed that, at some point in their development, most

*martinezordazm@gmail.com. 
scientific theories were thought to be inconsistent or falsified or incompatible with the best theories from other domains. Scientists kept working with such theories nonetheless. Some of the most famous examples of this are: Aristotle's theory of motion, the early calculus, Bohr's theory of the atom and Classical Electrodynamics, among others. This has given the impression that contradictions are not as malign as they were initially portrayed. Sometimes, contradictions can be tolerated and scientists can safely trust two mutually inconsistent statements.

The tolerant attitude towards contradictions is called "inconsistency toleration" and it is practiced when agents reason sensibly from inconsistent information without threatening their rationality. Inconsistency toleration is often seen as the result of combining the scientists' ignorance with the use of special inferential mechanisms that preserve sensible reasoning in inconsistent contexts. The former has been mostly described in terms of "factual ignorance" and the latter has been explained by appealing to different paraconsistent $\operatorname{logics}{ }^{1}$ In sum, in cases of inconsistency toleration, ignorance is the first to occur and it is followed by the avoidance of logical triviality.

Here I contend that ignorance plays a privileged role for the tolerance of contradictions in the empirical sciences. I argue that, at least, two types of ignorance are present when scientists adopt a tolerant attitude towards contradictions in the sciences: factual ignorance and ignorance of theoretical structure.

The plan for the paper goes as follows. In Sec. 2, I discuss the philosophical explanations that relate inconsistency toleration to ignorance. Later on, in Sec. 3, I scrutinize the relation between inconsistency toleration and factual ignorance, and in Sec. 4 I argue that factual ignorance is not sufficiently explanatory of interesting cases of inconsistency toleration, and, more importantly, that inconsistency toleration might be caused by other type of ignorance, ignorance of theoretical structure. In Sec. 5. I present a case study from neutrino physics that illustrates the role that ignorance of theoretical structure plays when explaining under which circumstances it is rational to tolerate contradictions in the empirical sciences. In Sec. 6. I explain how to move from factual ignorance to inconsistency toleration. Finally, Sec. 7 is devoted to drawing some conclusions on the connections between ignorance and inconsistency toleration.

\section{The generic explanation}

Once a scientist recognizes a contradiction in a set of relevant information, she can either reject such a set or keep working with it despite its inconsistency. If doing the former, she would need to explain why she dismissed any of the propositions involved - for instance, if it is known that at least one of them

\footnotetext{
${ }^{1} \mathrm{~A}$ logic is paraconsistent if it invalidates the Principle of Explosion and allows the avoidance of logical triviality despite the use of inconsistent information. A logic $L$ is paraconsistent if the $L$-closure of some inconsistent premise sets is non-trivial.

Some of the formal resources that resulted from the development of paraconsistent logics have been used to describe and explain historical episodes that illustrate both the presence of contradictions in the sciences and the absence of logical triviality.
} 
is false, mistaken or vague. If choosing the latter, she would need to identify specific inferential mechanisms that allow her to work with inconsistent information and still avoid logical triviality - regardless whether these mechanisms help her to get rid of the contradiction in the long run. When succeeding at doing the above, the result is called "inconsistency toleration".

If inconsistency toleration is a rational practice, philosophers should explain: (i) under which circumstances scientists would be rationally inclined to tolerate a contradiction and, (ii) how they could preserve sensible reasoning when using inconsistent information. Nowadays there is still no common agreement among philosophers and logicians on which are the best ways to achieve (ii); however, they have largely agreed on the fact that ignorance plays an important role for determining when scientists can be rationally inclined to work with inconsistent information. Here I explore what this consensus consists of.

When explaining (i) philosophers generally appeal to the following:

1. When having two scientific statements that contradict each other, scientists tend to assume that, at least, one of them is false (Laudan, 1977: $56)$.

2. If scientists are able to distinguish which of the conflicting propositions should be regarded as false - due to being part of idealizations, fictions, among others-, then they would be able to explain how they could satisfactorily work on seemingly false information.

3. However, most of the time, when confronted with an inconsistent set of information, scientists ignore, at least, which of the mutually contradictory statements should be regarded as false (cf. Bueno, 1997, 2006; Brown, 1990; Priest, 2002).

4. Once this ignorance is acknowledged, if scientists have no better alternative to the inconsistent set of propositions, the toleration of the contradiction becomes the only option at hand - such a tolerant attitude towards contradictions is often seen by scientists as a temporary resource.

Call this the generic explanation.

The morale of this explanation is that inconsistency toleration and ignorance are closely intertwined. The common agreement on this connection is that ignorance plays an important role in motivating scientists to tolerate certain contradictions, but also, that it is a constitutive element of the temporary character of such toleration. This comes quite intuitively: if scientists tolerate a contradiction because they ignore the truth values of the conflicting propositions, but they are also aware of the negative connotation of inconsistency in the sciences, this tolerant attitude would change once the relevant information is acquired and a more (epistemically) virtuous body of information is obtained.

If the relation between inconsistency toleration and ignorance is the one suggested by the generic explanation, philosophers should still address how scientists reach the point in which, when faced with a contradiction, they recognize to ignore which of the mutually contradictory propositions is false. 
For the case of the empirical sciences, to respond to this challenge does not seem difficult. As empirical sciences involve, most of the time, the use of incomplete information, it does not come as a surprise that scientific theories lack, at different points in their development, important epistemic virtues, such as consistency. Neither should it surprise us that, in the majority of cases, when scientists are faced with a contradiction, they lack the - either conceptual, experimental or mathematical- resources for determining which of the conflicting propositions should be regarded as false, even if they consider that it is impossible for both propositions to be true (see Bueno, 2006; Martínez-Ordaz, 2017).

\subsection{Some objections}

A challenge that the generic explanation might face is the following: given the commitments to classical logic that this explanation has, ignorance can equally justify either the rejection or the toleration of a specific contradiction 2 In addition, inconsistency toleration can be equally seen as an indicator of either prior ignorance or factual knowledge of true contradictions. This considered, the connection between ignorance and inconsistency toleration might not be as legitimate as the generic explanation assumes.

First, if one grants that contradictions are always false (as expressed in 1), once a scientist identifies a contradiction, she would know immediately that any set of information that contains it will be false - even if she ignores the individual values of the conflicting propositions. If this were the case, then she would be justified to reject such a set. Therefore, because of 1 , there is no need for toleration.

A way to respond to this first objection is to say that rejection is an option only if there is a consistent alternative for the inconsistent set of information. In those cases, the most rational decision would be to reject the inconsistent set and to work only with the consistent alternative. Nonetheless, if there is no alternative, scientists face the dilemma of either getting rid of both statements and ending up empty-handed or tolerating the contradiction pro tem-while acquiring the needed information for solving the conflict. In this case, it is less rational to leave scientists unarmed than to request them to find ways to satisfactorily reason with the inconsistent set of information (an argument for similar scenarios could be found in [Lakatos, 1978: Cap. 1, Sec. 2.b]) $]^{3}$

A resulting extremely well-spread consideration is that, if not having a consistent substitute for the inconsistent set, rejection should not be an option. As

\footnotetext{
${ }^{2}$ These commitments to classical logic include, at least, the spirit of bivalence that permeates the whole argument and the assumption of contradictions being false (as expressed in 1).

${ }^{3}$ Lakatos understood, in a very different way from Popper and Hempel, that the role of contradictions in scientific dynamics is different from simply refuting hypotheses or justifying a simplistic form of theory rejection. As a matter of fact, not only research programs often proceed legitimately in an ocean of anomalies, but also to be tolerant towards the presence of anomalies is a decisive factor of progress in mathematics.

I thank an anonymous referee for giving a better phrasing to my ideas on this point.
} 
a matter of fact, the most rational decision in these cases is to tolerate the contradiction while, at least, filling up the blanks (see Batens, 1998, 2002; Meheus, 2002; Bueno, 2017).

Second, let's consider the possibility of contradictions being true: if contradictions are true they can be the object of knowledge. Therefore, ignorance and inconsistency toleration would not be related at all.

In philosophical logic the possibility of true contradictions has been largely addressed by a standpoint called "dialetheism". Dialetheism is the view according to which some contradictions are true. Dialetheists might disagree on both premise 1 of the generic explanation and the temporary character of inconsistency toleration - specially in the formal sciences. Nonetheless, the main problem for dialetheism has been to determine which are those exceptional contradictions that can be true. On this issue, even dialetheists agree on the fact that if true contradictions in the formal sciences are already extremely rare, they would be even more so in the empirical realm (see Priest, 1998: 423). As a matter of fact, the majority of contradictions that emerge in the empirical sciences are not true and this is why they are only temporarily tolerated until scientists find a consistent alternative (see Priest, 1998, 2002) ${ }^{4}$ As a result, even if dialetheism has been very useful for dealing with semantic paradoxes, it has severely struggled at finding exemplars of true contradictions in the empirical sciences. The morale is that the mere possibility of a specific contradiction being true is not enough for justifying inconsistency toleration in the empirical sciences. This considered, true contradictions do not pose a real challenge against the generic explanation.

Third, according to the generic explanation, if committed to classical intuitions (bivalence in particular), one would accept the following: (a) if $p$ is the case, $\neg p$ cannot be the case and vice versa and (b) always, either $p$ is the case or $\neg p$ is the case. This gives the impression that, if it were possible to determine the truth value for either $p$ or $\neg p$, one could immediately infer the truth value of the other proposition.

In light of this, the reader might challenge the adoption of bivalence in cases in which scientists face contradictions; however, even if rejecting bivalence, the profits associated to knowing which of the conflicting propositions is false remain undeniable. To determine that $p$ is false can allow scientists to either get rid of it or look for explanations of why such a proposition can be satisfactorily used in specific contexts despite its falsity - such explanations would often appeal to the epistemic benefits of using abstractions, idealizations and fictions in the sciences

\footnotetext{
${ }^{4}$ The most compelling exemplar of a dialetheistic empirical theory is the Hegelian theory of motion discussed in [Priest, 1987]. This theory says that, in light of Zeno's paradoxes, contradictions are necessary for explaining two important physical phenomena: the nature of the instant of change and the nature of motion -change of location with respect to time (Priest, 1987: 165,166).

According to Priest, in this case, contradictions are both explanatory and the best option that scientists had at hand. However, Boccardi and Macías-Bustos (2017) have recently argued that contradictions are dispensable when developing a successful theory of motion and that a Russellian account, a consistent available alternative, is more explanatory than the Hegelian theory. Therefore, not even for this exemplar, one should grant contradictions to be true.
} 
(on this subject see [Pritchard, 2016]). Therefore, as the generic explanation expresses, to ignore the truth value of each of the conflicting propositions is vital for the toleration of contradictions in the sciences.

From the outset I want to be clear about the dialectic. None of the philosophers who hold that ignorance does an important work when explaining inconsistency toleration would content that ignorance in itself suffices for justifying the tolerance of contradictions in the sciences. Ignorance is the first to occur and it justifies the rational inclination for tolerating a specific contradiction, but once an agent feels inclined to do so, she still has to identify a way to avoid logical triviality when working with inconsistent information ${ }^{5}$

Given the close relation between ignorance and inconsistency toleration, the study of the types of ignorance present when dealing with contradictions in the sciences can shed light on the different ways in which scientists can tolerate such contradictions. My aim in the following section is to deepen into the type of ignorance that is described by the generic explanation.

\section{$3 \quad$ Factual ignorance and contradictions}

When faced with a contradiction, if scientists were aware of which of the conflicting propositions is false, they would be justified for dismissing it immediately. Nonetheless, if ignoring this, they lack justification for getting rid of any of the conflicting propositions. In absence of consistent alternatives, the best option at hand is to tolerate the contradiction -at least, temporarily. This kind of ignorance is what has been called by epistemologists "factual ignorance". Here I discuss how factual ignorance takes place in scenarios of inconsistent science.

\subsection{Factual ignorance}

I start with some preliminaries.

First, there are two rival accounts of what ignorance could be. "On the first view, called the 'Standard View', ignorance is lack or absence of knowledge, whereas on the second view, called the 'New View', ignorance is lack or absence of true belief" (Le Morvan and Peels, 2016: 12) -here I assume the former.

Ignorance understood as the absence of knowledge could be divided into three subcategories: absence of factual knowledge (lacking knowledge of either facts or the truth of specific propositions), absence of objectual knowledge (not knowing a particular object) and absence of procedural knowledge (not knowing how to do certain tasks) ${ }^{6}$ As inconsistency toleration is often assumed to be

\footnotetext{
${ }^{5} \mathrm{I}$ am greatly indebted to Otávio Bueno and the referees for helping me to give a better phrasing to my ideas here.

${ }^{6}$ In recent years, another type of ignorance has been put forward: absence of answers to questions, which has been commonly labeled as "erotetic ignorance". Nonetheless, as this type of ignorance remains very much unexplored, there is no clarity regarding its status compared to the other three subcategories; in particular, nowadays there is an open debate on whether erotetic ignorance reduces to factual ignorance. Here I do not focus on erotetic ignorance; however, for more detailed analyses on the subject see: [Rescher, 2009], [Nottelmann, 2016]
} 
motivated by scientists ignoring the truth values of the conflicting propositions, in the rest of this section, I focus exclusively on factual ignorance.

Factual ignorance can be the result of epistemic agent's failure at fulfilling any of the basic conditions for factual knowledge. Namely:

(i) a doxastic condition: $S$ believes that $p$;

(ii) an alethic condition: $p$ is true;

(iii) a justificatory condition: $S$ believes that $p$ with justification;

(iv) a Gettier-proofing condition: $S$ 's justification for believing that $p$ must withstand Gettier-type counterexamples. (Le Morvan and Peels, 2016:18).

For the purposes of this paper, I focus exclusively on the factual ignorance that results from the non-satisfaction of the alethic condition.

The alethic condition can only be satisfied if the content of a specific belief $p$ is true; nonetheless, there are different ways in which an epistemic agent $S$ can fail at satisfying this condition. The most common of them are:

- $S$ holds a false belief. $S$ can believe a proposition $p$ without being aware of $p$ being false and still reason sensibly from it. To believe something false can only prevent some one from knowing (if truth is a necessary condition for knowledge), but would not be sufficient for an agent to be irrational.

For instance, in the 19th Century, the French naturalist Félix Archimède Pouchet contended that life could be generated from non-related matter; dust could originate flies and parasitic worms could be generated from scratch by the human body itself. Despite believing false information, he was capable of reasoning sensibly when using false data, most of what he produced cannot be considered knowledge nowadays, but the arguments that he provided were not aleatory nor irrelevant. This is evidence of him being both rational and ignorant 7

- $S$ fails at assigning an alethic value to a specific belief. When believing $p, S$ cannot determine whether $p$ is the case or if she is mistaken. This type of ignorance is often not caused by the phenomenon itself but by a temporary lack of resources to test the truth of $p$.

For example, in the 18th Century, (early) parasitologists could not determine whether parasitic worms in the human body where generated solely by previously ingested parasitic worms or by the human body itself. This

and [Peliš, 2017].

${ }^{7}$ At the time, Pouchet was one of the most important supporters of the theory of heterogenesis, which was a variation of the theory of spontaneous generation, which contented that matter is capable of generating living organisms that do not need to be biologically related to each other, for example, new living organisms came from inanimate objects, such as air. Nowadays it is well known that this theory is false but in the early 19th Century it was still a candidate for explaining the origin of life. 
is, they could not decide the truth values of propositions such as $\ulcorner$ the presence of parasitic worms would cease by balancing the various body fluids in the body of an ill person $\urcorner$. Such a difficulty was not caused by the nature of the phenomenon itself, but by the lack of experimental techniques that scientists suffered at the moment.

- $S^{\prime}$ cognitive limitations prevent her from knowing a particular fact. $S$ holds a believe $p$ but the fact behind $p$ would be epistemically inaccessible to agents such as $S$.

An example of this type of ignorance could be

our knowledge of the universe we inhabit seems fundamentally restricted by the light-cone, i.e. the vast conical section of the universe (considered as Minkowski space-time), from which electromagnetic radiation travelling in vacuum could reach us. Certainly it seems plausible that there is a fact of the matter concerning the number of DNA molecules in the huge portion of the Universe outside the light-cone. Even if this fact is unknowable, certainly we are ignorant of it. (Nottelmann, 2016: 35)

In what follows, I focus on the second type and I explain its connection with inconsistency toleration.

\subsection{Ignorance as undecidability}

Going back to inconsistency toleration. When philosophers explain cases of inconsistency toleration they appeal to a specific type of factual ignorance, namely, the one caused by the agent's failure at assigning an alethic value to a specific proposition. Here, I briefly elaborate on how to understand factual ignorance for cases of inconsistency toleration.

In this sense, the alethic condition is satisfied if $S$ succeeds at assigning a unique truth value to $p$, this is, to determine whether $p$ is true. If the agent cannot assign values neither to $p$ nor to $\neg p$, the condition is not satisfied. If not satisfied this condition, the agent would be unable to reject or accept any of the two propositions in dispute. When the alethic condition is not satisfied, $S$ can either retain both statements meanwhile deciding the values of the contradiction or reject both statements a priori.

One of the worries that the reader might entertain regarding this view is the following: if characterizing ignorance as the complement of knowledge, the understanding of ignorance will be strongly (and problematically) tied to a specific conception of knowledge - which, very likely, would be still extremely contentious among philosophers.

With the above in mind, I suggest to see this type of factual ignorance to focus on the connections between ignorance and truth values from a philosophically (maybe) less contentious point of view. I understand this factual ignorance as the temporary undecidability of the truth value of a proposition $p$ by an epistemic agent $S$ at a specific time $T_{1}$. This 'undecidability' is not necessarily as 
perceived in the literature of logic and philosophy of mathematics -as for the cases of inconsistent empirical sciences the truth values in question are, in the long run, likely to be determined 8

The causes of this undecidability might be very diverse. As in the empirical sciences facts are mostly discovered via experimentation, if scientists lack experimental resources to test their hypotheses and predictions, this can prevent them from knowing whether the relevant propositions are true. In addition, some phenomena, like eclipses, once predicted, require time to pass before being tested and therefore, such facts remain ignored for a period of time.

When scientists are unable to assign truth values to two mutually conflicting hypotheses, this ignorance might lead them to either reject a priori one of the hypotheses, to get rid of the two of them or to keep working with both meanwhile discovering which one is false.

Summing up, according to the generic explanation, factual ignorance as temporary undecidability is often enough for making scientists to be rationally inclined to tolerate contradictions in the sciences. Furthermore, this ignorance is explanatory of the temporary character of such tolerant attitude towards contradictions.

In the following section, I challenge this view by arguing that while factual ignorance plays an extremely important role for motivating the tolerance of contradictions in science, what is at stake in cases of inconsistent science is often a more complex type of factual ignorance, namely, ignorance of theoretical structure.

\section{Ignorance of theoretical structure}

On the surface, factual ignorance as temporary undecidability could seem to be enough for explaining the rational tolerance of contradictions in science. Nonetheless, even if this impression is correct, philosophers still need to explain where this factual ignorance comes from.

I contend that, for many cases of inconsistency toleration, temporary undecidability is caused by a different type of ignorance, namely, ignorance of theoretical structure. In order to do so, I proceed as follows. First, I briefly introduce some conceptual specifications on the structure of scientific theories and holistic properties of bodies of scientific knowledge. Later on, I characterize ignorance of theoretical structure and explain how it motivates inconsistency toleration.

\subsection{Scientific theory}

Scientific theories are epistemic vehicles that help scientists to filter, order and relate the varied information that they get about the world in order to provide accurate descriptions, predictions, and explanations of a specific domain.

\footnotetext{
${ }^{8}$ If interested in the connections between this conception of undecidability for statements from the empirical sciences see [Gutiérrez-Ramírez, 2015: Chap. 1. In Spanish].
} 
Theories are often considered to be clusters of information which are initially incomplete but that, in the long run, tend to incorporate new data in order to improve the picture of the world that they provide. 'The world' itself is an infinitely large source of information, and one "of the most obvious sources of ignorance is the sheer volume of available factual information. There is so much out there to be known that any given individual cannot ever begin to make more than an insignificant fraction of it" (Rescher, 2009: 4).

Let scientific theories to be formulated based on the following set-theoretic construct: $T=<\boldsymbol{D}, \boldsymbol{R}_{i}^{n}>$ "where $\boldsymbol{D}$ is a particular domain (a set of objects to which the theory is supposed to apply) and $\boldsymbol{R}_{i}$ is a family of $n$-place relations holding between the elements of $\boldsymbol{D}$ " (Bueno, 1997: 588). $T$ consists of a set of substructures (partial structures), $\left\langle A, A^{\prime}, \ldots A^{n}\right\rangle$, of the form $A=<D$, $R_{k}>_{k \in K}$. Where $R_{k}$ are partial relations ${ }^{9}$ so that $R_{k}=<R_{k 1}, R_{k 2}, R_{k 3}>$, and $R_{k 1}, R_{k 2}$ and $R_{k 3}$ are mutually disjoint sets such that:

- $R_{k 1}$ is the set of $n$-tuples that (we take to) belong to $R$,

- $R_{k 2}$ is the set of $n$-tuples that (we take) do not belong to $R$,

- $R_{k 3}$ is the set of $n$-tuples for which it is not defined whether belong or not to R. (Bueno and French, 2011: 858-59).

The domain, $D$, could be selected and individuated depending on the methodological preference of the research program in which the theory is being used and vary from time to time. The relations, $\boldsymbol{R}_{i}$, help to order, classify, and evaluate the objects in the domain - and the propositions through which they are described. The relations between the objects of $\boldsymbol{D}$ are closed under specific logical consequence relations, allowing and forbidding certain interactions between them.

An inconsistent theory contains both a sentence $s$ (taken to be, at least, quasi-true) as well as a sentence $\neg s$ (also taken to be, at least, quasi-true).

it should be noted that quasi-truth is strictly weaker than truth, in the sense that if a sentence is true, then it is quasi-true, but the converse doesn't hold in general. Moreover, if a sentence is quasifalse, then it is false, but the converse doesn't hold in general either. (For further details, see Bueno [2000], and da Costa and French [2003].) Furthermore, it is also possible that a sentence $s$ is quasitrue and its negation, $\neg s$, is quasi-true as well. (Bueno and French, 2011: 860)

This approach to scientific theories is called "Partial Structures approach", and it has proved to be extremely handy when explaining and modeling inconsistency in the empirical sciences (see Bueno, 1997; da Costa and French, 2003; Bueno and French, 2011).

\footnotetext{
${ }^{9} \mathrm{~A}$ partial relation $R_{i}$ over $D$ is a relation that is not necessarily defined for all $n$-tuples of elements of $D$ (see da Costa and French 1990: 255).
} 
The basic idea behind Partial Structures is that science is essentially an open endeavor and because of that, systematically, scientists work with partial, conflicting and inconsistent information. Partial structures account for the defective theories that result from scientists working with defective (in particular, partial) information in their day to day practice, submitting that such theories are at least partially true with respect to a specific domain. The partial structures' characterization of scientific theories leaves room for the ways in which the partiality of information and ignorance interact in the scientific endeavor without putting in danger scientific rationality; as a matter of fact, the $R_{3}$ component allows us to address this issue by hosting the elements that have not yet been determined to be models of the theory.

\subsection{Theoretical structure}

Scientific theories relate varied types of data and they do so in a very special way: they follow and preserve privileged inferences that are considered to be linked to the theory's predictive and explanatory success. What scientists

really study are not any objects and their properties, but certain general inference relations or inference patterns (...) What exactly does speaking of 'inference relations' here involve; in particular, what are the relata: mere sentences (so that we are back to some kind of formalism?), propositions (leading us beyond formalism after all?), etc.? (Reck and Price, 2000: 347-48)

According to this view, one of the main tasks of scientific theories is to preserve and stress particular inference patterns between propositions -and it is expected that such patterns warrant the success of the theory in different contexts 10

The identification of reliable inference patterns is one of the most important scientific tasks. To identify inference (logical) strategies for the preservation of the success within our best theories is closely related to the preservation of (epistemic) reliability. That said, it is important to state that this does not imply that scientific theories are structures, but that structures constitute a useful "mode of representation' and it is important to reiterate that, on my view, adopting it does not entail that either theories or the structures they put forward as 'out there' in the world should be regarded as inherently set-theoretic in any way" (French, 2012: 5).

Inference patterns determine largely the type of results that scientists can get in their day to day use of theories. For instance, there are historical cases in which the chosen set of patterns restrict the use of conjunction as a reliable inference (see Brown and Priest 2004, 2015), or cases where reductio ad absurdum-type of proofs were regarded as not reliable (see Meheus, 2002).

Now, let a structure be a set of operations and relations between collections of objects such that, in a certain way, has the epistemic role of facilitating the study

\footnotetext{
${ }^{10}$ If $\ulcorner$ All $P$ are $Q\urcorner$ is derivable from the theory, then to derive $\ulcorner Q a\urcorner$ from $\ulcorner P a\urcorner$ is an inference pattern.
} 
of the objects contained by the collections. For the case of the empirical sciences, a structure cannot be "construed as 'pure' logico-mathematical structure (see French, 2007); it was always intended to be understood as theoretically informed structure" (French, 2012: 7), this is, structures that were constantly informed by the (empirical) commitments associated to the domain of application of the theories in question.

Let theoretical structure be the (inferential) structure of empirical scientific theories.

\subsection{Knowledge of theoretical structure}

Can scientists come to fully know the theoretical structure of a particular theory? Due to both our cognitive limitations and the complexity of our empirical theories, this might be just impossible. Yet, this does not mean that scientists cannot know some important parts of the theoretical structure of their theories. As a matter of fact, to know certain parts of the theoretical structure of her theories gives the scientist the capability of knowing which inferences are correct in a particular context and also why such inferences have certain specific consequences.

When scientists master specific inference patterns within a particular domain, what they gain is a way to structure and follow successfully certain inferences in their day-to-day practice; this is, not only that they can use inferential rules in an effective way but also that they can explain under which circumstances and why certain inferential rules are reliable in a domain of application of their theory.

Knowledge of theoretical structure is not a simple case of factual knowledge, as to say that someone knows certain structure does not mean only that that person knows the truth value of specific propositions. Indeed, to know which inferences are allowed within a theory can help scientists to determine the truth value of propositions of the theory, but it can also provide, for example, important hints on the logical principles that govern that segment of the theory. So, while knowledge of a fragment of a theoretical structure allows agents to achieve knowledge of the truth values of specific propositions that are located in such a fragment, knowledge of theoretical structure seems to be something deeper.

A clear way to emphasize the differences between a simple case of factual knowledge and factual knowledge associated to a theoretical structure would be to describe the way in which these two kinds of knowledge can fail. The impossibility of determining the truth values of certain propositions in the empirical sciences, could be simply associated, for instance, to a lack of experimental resources. For example, still in the 19th Century, early parasitologists lacked experimental methodologies sophisticated enough to test the origin of parasitic worms, but this was not really caused by the structure of any of the theories involved nor by the ultimate nature of the phenomenon, but only by a lack of experimental development.

Nonetheless, sometimes, the scientists' incapability of determining the truth value of certain propositions within a theory is caused by their lack of epistemic 
access to the structure of the theory. This can occur if scientists have not figured out how certain parts of the theory hang together and which are the adequate inferences that could be ran in that segment of the theory. Such a lack of information explains why inconsistency toleration tends to last for long periods, this is until scientists acquire knowledge of the relevant inference patterns of their theories.

Knowledge of theoretical structure can sometimes get mistaken for the agents' ability to follow inferences within a theory (a sort of procedural knowledge). However, while procedural knowledge of inference maneuvers might result from knowing the theory's inference patterns, knowledge of theoretical structure is a sophisticated kind of factual knowledge. Knowledge of theoretical structure is not only about being competent when using a theory, it is about determining if certain propositions are true within the theory.

\subsection{Ignorance of theoretical structure}

If what has been said here is along the right lines, I propose to characterize the ignorance of theoretical structure in the following way.

Ignorance of theoretical structure: absence of knowledge regarding the (relevant) inference patterns that scientific theories allow for. When ignoring (the relevant parts of) the theoretical structure of a theory, scientists are not capable of grasping abstract causal connections between the propositions of their theory, they can neither identify the logical consequences of the propositions that they are working with nor can explain under which conditions the truth value of such propositions will be false.

Ignoring the inferential patterns that the theory allows for can prevent scientists from assigning truth values to some propositions associated to the theory. At the beginning, this might be mistaken as a case of just temporary undecidability; yet, if the problem remains for considerable long time, even after the development of new experimental and theoretical resources, this ignorance might being caused by something deeper, namely, ignorance of theoretical structure.

But, what gives rise to ignorance of theoretical structure? This type of ignorance is often linked to the early stages of scientific theories. It is quite intuitive that if a theory is too young, and still under development, scientists will be exploring the inferential patterns that the theory allows for without having certainty of what constitutes a theorem of the theory. Another possible cause of this kind of ignorance might be the holistic character of bodies of scientific knowledge plus lack of epistemic access to the structure of the theory. If the degree of internal or external holism, is extremely high, scientists would not be able to identify privileged inferential relations that allow them to test the truth value of the involved premises, among other things. 


\section{$5 \quad$ Ignoring the theoretical structure of neutrinos}

This section is devoted to provide a case study that illustrates the close relation between ignorance of theoretical structure and inconsistency toleration. In a nutshell: the following case study describes the way in which neutrino physicists tolerated a contradiction for almost three decades, mainly because they ignored the way in which the elements of their theories related to each other - which prevented them from isolating the contradiction.

\subsection{The story}

For many years, neutrinos were theoretical entities that help to explain and predict nuclear reactions -which later would be known as " $\beta$-decay". In 1960, physicists finally believed that it was possible to design an experiment to prove the existence of neutrinos -this, through the measuring of the flux of solar neutrinos ${ }^{11}$ In order to do so, physicists combined information from different disciplines -which included radiochemistry, nuclear physics, astrophysics, and neutrino physics (Pinch, 1986: 47). With that information put together, physicists were able to come up with what was needed in order to test the existence of solar neutrinos. On the one hand, John Bahcall designed a mathematical model that helped to predict the flux of solar neutrinos, this model was named 'Standard Solar Model' (henceforth, SSM) (see Bahcall, 2003: 78) 12 On the other hand, Ray Davis was expected to design an experiment to tests the predictions of the SSM. The experiment made use of the combination of knowledge from radiochemistry, neutrino physics, nuclear physics, among other areas of physics, as well as of complex apparatus and instruments -as a super cooled underground tank and a Geiger counter.

When, in 1967, Davis did the experiment, the results showed that the SSM's predictions were 2.5 times larger than the results reported by Davis (see Bahcall, 2003: 79). Davis blamed Bahcall's calculations, and Bahcall attributed the conflict to the experiment. Both research groups spend the year of 1968 reviewing and correcting the $S S M$ and the setup for the experiment. After almost a year, they ran the experiment once again, but the results were not yet satisfactorily. The difference between the predictions and the observational results was still large enough to be explained by appealing to a margin of error;

\footnotetext{
${ }^{11}$ Solar Neutrinos are subatomic particles that are generated from solar fusion; it was believed, that this type of particles did have neither electric charge nor mass.

${ }^{12}$ The $S S M$ is a

theoretical framework derived from the application of laws about energy conservation and transport(...) The $S S M$ consists of a set of assumptions both theoretical and empirical, that -depending on the interpretation of the $S S M$ that is used- could efficiently describe a unique empirical domain, in this case, the Sun. It has also the capability of giving descriptions of specific phenomena, predictions and guidance for experiments on the phenomena it describes, one its applications is to describe and allow to make predictions regarding the flux of solar neutrinos. (Martínez-Ordaz, 2017: 133.)
} 
this made impossible to consider the observational outcome as evidence in favor of the SSM. The problem remained unsolved until 2001 when it was discovered that neutrinos are of different types and that they have mass; whit this, it was clear that to ignore those facts was what originated the anomaly regarding the measuring of their flux.

Since it was very difficult for the scientific community to point out where the inconsistency originated -they did not agree for a long time which part of which theory had to be modified-, it was impossible for them to satisfactorily isolate the problematic part(s) of the theory. But this did not mean that they stopped using the SSM nor relying on some of the basic assumptions of the theories involved in the design of the experiment. Indeed, they kept the theory in use and they continued experimenting with the solar neutrinos phenomena, as the reports from the Kamiokande, the SAGE, the GALLEX, and SuperKamiokande could show (see Bahcall and Davis Jr., 1976; Bahcall, 1981; Pinch, 1986; Franklin, 2003). I consider this to be enough to say that scientists kept using the conflicting assumptions despite the discovery of the inconsistency, which could only be understood as if they were tolerating the inconsistency ${ }^{13}$

There was a common agreement among the involved physicists on the fact that ignorance played an important role when explaining why it took so long to resolve the contradiction (see Pinch, 1986), it is not clear that the involved scientists actually possessed factual ignorance. First, the major problem was that, at the moment, they ignored many of the inferential relations that the $S S M$ allowed for as well as many of the inferential relations that connected the theories that underlie the experimental design. Nonetheless, it was not clear that they had no epistemic access to the truth value of the majority of propositions that they considered of interest.

\subsection{Explaining the case study}

When physicists were dealing with the anomaly in the measuring of the solar neutrinos' flux, it was almost impossible for the scientists to identify which were actually the conflicting statements. While the explicit contradiction was between the predictions and the observational reports, it was thought that the actual conflict was located within the theories - the Standard Solar Model, $S S M$, and the theory that underlay the experiment.

Take the $S S M$ to be formulated as follows: $S S M=<\boldsymbol{D}, \boldsymbol{R}_{i}^{n}>$, for which,

(a) $\boldsymbol{D}$ consists on a set of compositional properties of the stars.

(b) $R_{i}$ is a family of $n$-place relations holding between the elements of $\boldsymbol{D}$.

(c) $S S M$ consists of the set substructures (partial structures):

\footnotetext{
${ }^{13}$ For a detailed analysis of this particular case study as an exemplar of inconsistency toleration see [Martínez-Ordaz, 2017].
} 


$$
<A_{\text {NeuProd }}, A_{\text {NeuDetc }}, A_{\text {Heliosmg }}, A_{\text {RadiatvSp } \ldots A^{n}>14}
$$

(d) Partial structures are of the form: $A=<D, R_{i}>_{i} \in I$, for which, $D \subseteq \boldsymbol{D}$ and $R_{i}=\boldsymbol{R}_{i} \cap D$, where:

- $R_{N e u P r o d 1}$ contains only the elements of $D_{N e u P r o d}$ which fully satisfy a model of the theory with respect of the production of solar neutrinos,

- $R_{N e u P r o d 2}$ contains only the elements of $D_{N e u P r o d}$ that are known to not be models of the theory,

- $R_{N e u P r o d 3}$ contains only the elements which is not yet known whether they are models of $D_{\text {NeuProd }}$.

The anomaly in the measuring of the solar neutrinos' flux required to pay attention to at least four partial structures: $A_{\text {NeuProd }}, A_{\text {NeuDetc }}, A_{\text {NeuExp }}$ and $A_{\text {Emp }}$.

$A_{N e u P r o d}$, contains the $S S M$ elements associated to the production of neutrinos ${ }^{15} A_{N e u D e t c}$ contains the $S S M$ elements which are used for the detection and measurement of neutrinos -including the assumption that neutrinos were massless and of just one type. $A_{N e u E x p}$ includes the $S S M$ elements used for the experimental design - the majority of elements contained here were, significantly, shared by $A_{N e u P r o d}$ and $A_{N e u D e t c}$. Finally, $A_{E m p}$ contains the empirical elements of the $S S M$.

When the anomaly was discovered, scientists faced the following dilemma: either to weaken their commitments towards the empirical substructure $A_{E m p}$ or to weaken their commitments towards $A_{\text {NeuProd }}, A_{\text {NeuDetc }}$ and $A_{\text {NeuExp }}$.

On the one hand, the first option was ruled out by the $S S M$ 's success in other areas of research. As a matter of fact, $S S M$ was considered to be empirically adequate with respect to the prediction of the age of the stars, the calculations of luminosity and the presence of heavy metals in the stars, among other domains of application.

On the other hand, the second option was the most natural to take by the physicists. From 1968 to the end of the 1990's, the physicists' doxastic commitments towards some of the (scrutinized) assumptions of $S S M$ were weakened. Such assumptions include the information about cross sections of $A r^{37}$ and $C l^{37}$ (contained in $A_{\text {NeuProd }}$ ) and the problems linked to the calculation of Coulomb coefficients (contained in $A_{\text {NeuDetc }}$ ). The fact that, for long time, scientists

\footnotetext{
${ }^{14}$ Which are determined by the domain of application of the theory, for instance: while $A_{N e u P r o d}$ is constructed by taking all elements from the $S S M$ that were needed to predict the production of solar neutrinos, $A_{\text {Heliosmg }}$ is build by taking all $S S M$ elements that are needed for the study of wave oscillations in the stars. These substructures can shared elements between them but what does large part of the work is that they also have non-shared elements.

${ }^{15}$ This is, information about the process in which four protons are combined to produce two protons, two neutrons, two positrons; information about nuclear reactions and cross sections of elements such as $A r^{37}$ and $C l^{37}$, among others.
} 
were agnostic about these facts suggests that, from a partial structures approach, these elements would be naturally captured by the $R_{3}$ components -as their truth value within the theory was still to be determined.

Yet, the scientists' weakening of their doxastic commitments towards these facts was not enough to prevent the theory from being inconsistent. As a matter of fact, despite the commitments that scientists held towards the distinct sets of information, the conflicting predictions and observational reports were still reached, this is:

1. $S S M:<A_{\text {NeuProd }}, A_{\text {NeuDetc }}, A_{\text {NeuExp }}, A_{E m p}, \ldots, A^{n}>$

2. $A_{\text {NeuProd }} \vDash s$

3. $A_{\text {NeuDetc }} \vDash s$

4. $A_{\text {NeuExp }} \vDash s$

5. $A_{E m p} \vDash \neg s$

The above supports two main theses: first, it shows that scientists responded immediately to the presence of the contradiction by weakening their doxastic commitments. Second, it also shows that the weakening of the commitments is not enough for dissolving the contradiction; the theory was still inconsistent regardless what scientists thought about it, this is why they kept intensively trying to solve the conflict.

Since 1960, the traditional characterization of neutrinos as massless was taken to be more an empirical constraint than a theoretical one. Therefore, this assumption was everywhere, it was shared by all the substructures that addressed the problems associated to neutrinos' presence. However, by the end of the 1990's, when the hypotheses of neutrinos being of different types as having mass were seriously considered, these assumptions (included in the three relevant substructures, $A_{\text {NeuProd }}, A_{\text {NeuDetc }}$ and $A_{\text {NeuExp }}$, and the empirical substructure $A_{E m p}$ ) were also moved into the $R_{3}$ components.

By 2015, another strong change hit the theory, the assumptions of neutrinos being of different types as having mass were seriously considered were discovered to be true; this had the effect of moving the traditional characterization of neutrinos (as massless and of just one type) to the $R_{2}$ components. Finally, roughly speaking, the characterization of neutrinos provided by Takaaki Kajita and Arthur B. McDonald in 2015 is now quasi-true in the partial structures $A_{\text {NeuProd }}, A_{\text {NeuDetc }}, A_{\text {NeuExp }}$ and $A_{E m p}$. Doing so changed the landscape to the following:

1. $S S M:<A_{\text {NeuProd }}, A_{\text {NeuDetc }}, A_{\text {NeuExp }}, A_{\text {Emp }}, \ldots, A^{n}>$

2. $A_{\text {NeuProd }} \vDash \neg s, A_{\text {NeuProd }} \not \models s$

3. $A_{\text {NeuDetc }} \vDash \neg s, A_{\text {NeuDetc }} \not \models s$

4. $A_{\text {NeuExp }} \vDash \neg s, A_{\text {NeuExp }} \not \models s$

5. $A_{E m p} \vDash \neg s$

The above does not mean that the theory is finally completed, but that the $S S M$ is now regarded as empirically adequate with respect of measuring the solar neutrinos' flux.

At the moment, physicists ignored how all the different theoretical assumptions behind the $S S M$ and the experiment interacted. Given so, when the anomaly was discovered, they had to choose: either they rejected the $S S M$ and 
the experiment, or they entertained the conflicting theories while attaining a better understanding of the structure of the theories.

As the involved theories were independently used in other disciplines, it seemed irrational to lose confidence in them only because neutrino physicists had not yet achieved full understanding of how such theories related to one another. As a matter of fact, once scientists realized that they ignored the SSM's theoretical structure, they were inclined to tolerate the contradiction pro tem - until they surpassed their ignorance. Once knowledge of certain parts of the structure of the theories was achieved, physicists realized that the understanding of neutrinos required to take as false the hypotheses about neutrinos as massless and neutrinos being of just one type. To understand how these theories related allowed scientists to adequately determine the truth values of certain propositions.

This case study showed that, if scientists had sufficient insight of the structure of a theory, they can be justified for rejecting the conflicting propositions. This is, if they have known what was causing the contradiction, they could have cut-off the ill part of the theories without needing to work with an inconsistent set of information $\sqrt{16}$ Nonetheless, as they were ignorant of the cause of the contradiction, they were "forced" to continue reasoning with the theory despite its inconsistency. This happened because to carry out any satisfactorily revision of the elements contained in a theory, in order to locate the cause of the contradiction, requires insight of the theoretical structure, which they clearly lacked. When such an insight is not available and one is confronted with a problematic outcome, accepting the inconsistency might sometimes be the only way to continue practicing any science at all 17

In the next section, I tackle the issue of what else, besides ignorance, is needed for inconsistency toleration in the empirical sciences.

\section{From ignorance to toleration}

With the clarifications about factual ignorance in place, I want turn once more to the phenomenon of inconsistency toleration. I have explained that there are two types of factual ignorance associated to inconsistency toleration: temporary undecidability and ignorance of theoretical structure. While the former is often tied to the temporary lack of (experimental or theoretical) resources, the latter is absence of knowledge of the inference patterns that the theory allows for. I am now in a position to explain what it means to move from factual ignorance to inconsistency toleration.

In what follows, I explain why ignorance of theoretical structure is what connects ignorance to inconsistency toleration. The explanation that I provide here is ordered chronologically considering the most common way in which scientists

\footnotetext{
${ }^{16}$ Such a view is largely defended in [Davey, 2014] and a critical response to it is introduced in [Martínez-Ordaz, 2017].

${ }^{17}$ Thanks to the anonymous reviewer who helped me to give a better phrasing of my ideas on this point.
} 
go from the discovery of a contradiction to its toleration.

\section{- Ignorance of theoretical structure causes ignorance as temporary} undecidability.

When scientists face a contradiction, if they ignore the truth values of the mutually conflicting propositions, this ignorance is often caused by the fact that scientists also ignore the needed inferencial mechanisms to determine the values of such propositions. That is why, even when equipped with better experimental and theoretical resources, if the theoretical structure is still unknown, the contradiction remains unexplained, unsolved and dangerous.

\section{- Ignorance of theoretical structure motivates the chase for specific inference patterns.}

Scientists "do not simply respond to ignorance by leaving a mere blank. We have a natural a perfectly reasonable inclination to fill in those gaps in the easiest, most natural, and sometimes, even most attractive way" (Rescher, 2009: 2). This is, as factual ignorance alone can never suffice for justifying either inconsistency toleration or the rejection of the contradiction. Some further action is needed.

As a matter of fact, "the ignorant agent rarely (if ever) approaches the lack of information as a missing content, but she often permeates her cognition with possibilities and hypotheses that build a framework around that black space in order to justify or explain it" (Arfini, 2019: 28). Such frameworks can be seen as, at least, the identification, selection and organization of specific inference patterns that allow scientists to work safely with inconsistent information. The search of these patterns is motivated by the scientist's acknowledgement of her own ignorance regarding the privileged inferences within her theory -specifically, the inferences that would allow her to avoid logical triviality and still preserve the most valuable consequences of her theory.

\section{- Ignorance of theoretical structure guides the chase for specific inference patterns.}

When ignoring the theoretical structure of an inconsistent set of information, there would be different inference patterns to follow in order to preserve the desirable consequences of the theory and avoid logical triviality. Such patterns are classified as follows:

There are (A)consistency-preservation strategies and (B) inconsistency-toleration strategies(...)(a1) According to compartmentalization, domains of inquiry to which mutually inconsistent scientific theories apply are compartmentalized, with no overlap between them, and in this way classical logic is preserved (...) (a2) According to information restriction, a single domain 
is maintained throughout the sciences, and any resulting inconsistency is eliminated by (somehow) extracting conflicting information (...) Among the inconsistency-tolerant strategies: (b1) According to paraconsistent compartmentalization, a plurality of overlapping domains is allowed for throughout the sciences (...) (b2) According to dialetheism, all inconsistencies for which there is good evidence are taken to be part of a single scientific domain. (Bueno, 2017: 230)

These types of inference patterns often entail different ways of dealing with ignorance of theoretical structure. Some of them will give rise to scenarios in which an agent has acquired some knowledge about the inferences that allow her to preserve consistency while still entailing some desirable consequences of her theory; some others would allow her to discover ways to preserve some consequences of her theory while losing consistency and scope of the domain of application.

\section{- Inconsistency toleration is possible if and only if scientists over-} come their ignorance of theoretical structure.

The fact that the selection of particular inference patterns constitutes a necessary component of inconsistency toleration suggests that, regardless the cause of factual ignorance in every particular case, inconsistency toleration is evidence of the -at least partial- overcoming of ignorance of the relevant theoretical structure ${ }^{18}$ When a scientist works satisfactorily with inconsistency, she has acquired knowledge of some relevant parts of the theoretical structure.

Considering the above, the connection between ignorance of theoretical structure and inconsistency toleration is illustrated by the following diagram.

\footnotetext{
${ }^{18}$ Partial overcoming of ignorance of theoretical structure means that, when tolerating a contradiction, scientists need not to identify the ultimate or the total structure of their theory, but that they can provide a set of inference patterns that allow them to successfully use the theory in question while avoiding logical triviality.
} 


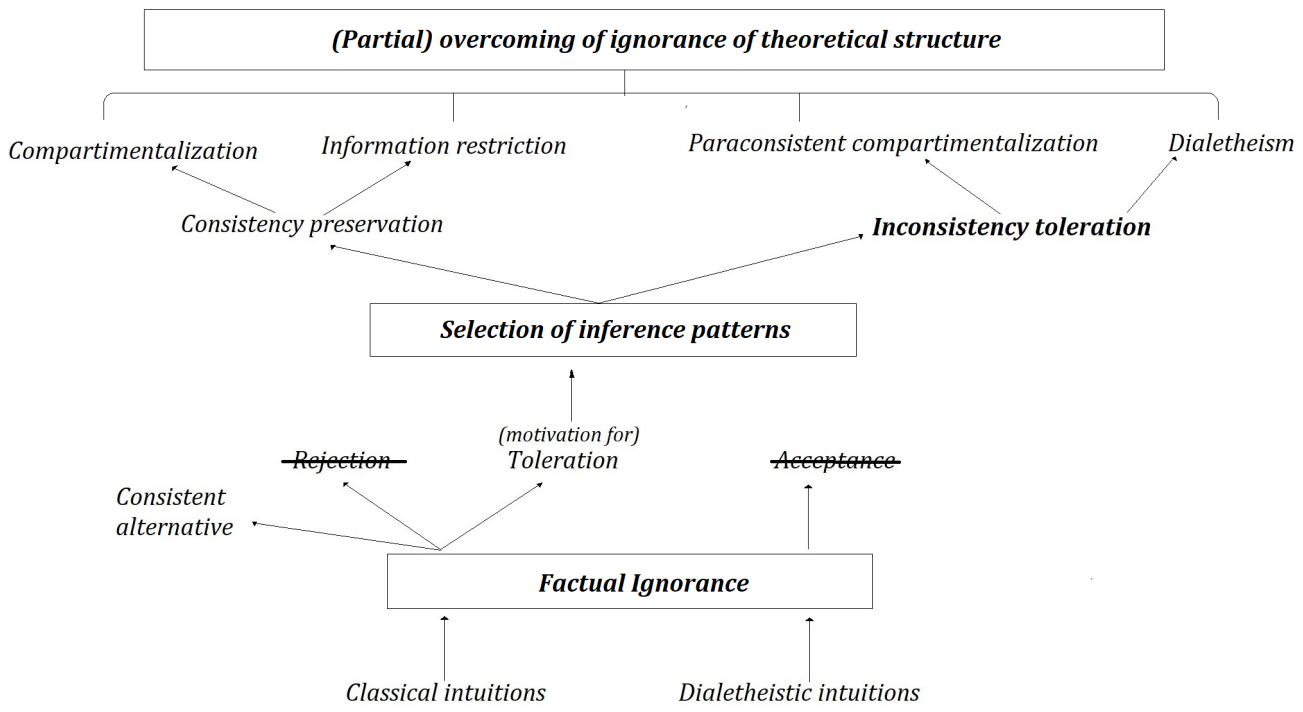

Figure 1. The general picture: ignorance and inconsistency toleration.

The lowest level of the diagram illustrates what has already been said in Sec. 2. when ignoring the values of the conflicting propositions, scientists can either abandon the inconsistent set of information and work with a consistent alternative (if available), or be inclined to tolerate the contradiction temporarily 19 The middle area shows that, if scientists identify concrete inference patterns to work with the inconsistent information, depending on the type of inferential maneuvers that they chose, they can either preserve consistency or tolerate inconsistency. The highest level shows that, if ignorance of theoretical structure is (at least, partially) overcome, inconsistency toleration becomes available to the scientists; and also if inconsistency toleration has been achieved, this indicates the partial overcoming of ignorance of theoretical structure.

In closing, I want to forestall any misconception, to tolerate a contradiction does not imply that there is no ignorance involved anymore. As a matter of fact, inconsistency toleration often shows that some knowledge of theoretical structure has been achieved, but while such a knowledge might be enough for the avoidance of logical triviality, it might still remain insufficient for determining whether $p$ or $\neg p$ are the case in the actual world.

\section{Concluding thoughts}

Here I explained the relation between inconsistency toleration and different types of ignorance. I argued that, when scientists find a contradiction in their

\footnotetext{
${ }^{19}$ Options such as simple rejection or (dialetheist) acceptance are ruled out for the reasons discussed in Sec. 2.1
} 
theories, if they recognize to be ignorant regarding either the truth values of the conflicting propositions or the structure of their theories, they can be rationally inclined to tolerate such a contradiction.

In order to do so, I proceeded as follows: in Sec. 2, I introduced the generic explanation of the relation between (factual) ignorance and inconsistency toleration. In Sec. 3, I explored different ways to understand and explain the presence of factual ignorance in the empirical sciences. Here I contended that, according to a large majority of philosophers, the ignorance that plays a prominent role in the majority of cases of inconsistency toleration is caused by the non-satisfaction of the alethic condition of knowledge. I characterized such ignorance as the (temporary) undecidability of the truth value of a proposition $p$ by an epistemic agent $S$.

Later on, in Sec. 4. I discussed a second type of ignorance, namely, ignorance of theoretical structure. Here I explained the way in which this type of ignorance is often what causes ignorance as temporary undecidability. Then, in Sec. 5 . I introduced a case study from neutrino physics, which illustrated ignorance of theoretical structure and the role that it plays for inconsistency toleration in the empirical sciences. Finally, in Sec. 6, I argued that, ignorance of theoretical structure accompanies in different senses, as well as at different moments, the toleration of contradictions in the sciences.

\section{References}

Arfini, S. (2019). Ignorant Cognition: A Philosophical Investigation of the Cognitive Features of Not Knowing. Studies in Applied Philosophy, Epistemology and Rational Ethics. Springer.

Bahcall, J. and Tremaine, S. (1981). Methods for determining the masses of spherical systems. i-test particles around a point mass. The Astrophysical Journal, 244:805-819.

Bahcall, J. N. (2003). Solar models: An historical overview. International Journal of Modern Physics A, 18(22):3761-3776.

Bahcall, J. N. and Davis, R. (1976). Solar neutrinos: a scientific puzzle. Science, 191(4224):264-267.

Batens, D. (1998). Paraconsistency and its relation to worldviews. Foundations of Science, 3(2):259-283.

Batens, D. (2002). In defence of a programme for handling inconsistencies. In Inconsistency in science, pages 129-150. Springer.

Brown, B. (1990). How to be realistic about inconsistency in science. Studies in History and Philosophy of Science Part A, 21(2):281-294. 
Brown, B. and Priest, G. (2004). Chunk and permeate, a paraconsistent inference strategy. part i: The infinitesimal calculus. Journal of Philosophical Logic, 33(4):379-388.

Brown, B. and Priest, G. (2015). Chunk and permeate ii: Bohr's hydrogen atom. European Journal for Philosophy of Science, 5(3):297-314.

Bueno, O. (1997). Empirical adequacy: A partial structures approach. Studies in History and Philosophy of Science Part A, 28(4):585-610.

Bueno, O. (2006). Why inconsistency is not hell: Making room for inconsistency in science. Knowledge and Inquiry: Essays on the Pragmatism of Isaac Levi, pages $70-86$.

Bueno, O. (2017). Scientific pluralism, consistency preservation, and inconsistency toleration. HUMANA. MENTE Journal of Philosophical Studies, 10(32):229-245.

Bueno, O. and French, S. (2011). How theories represent. The British Journal for the Philosophy of Science, 62(4):857-894.

da Costa, N. C. and French, S. (1990). The model-theoretic approach in the philosophy of science. Philosophy of Science, 57(2):248-265.

da Costa, N. C., Newton, C., da Costa, N. C., French, S., et al. (2003). Science and partial truth: A unitary approach to models and scientific reasoning. Oxford University Press on Demand.

Davey, K. (2014). Can good science be logically inconsistent? Synthese, 191(13):3009-3026.

Franklin, A. (2003). Are there really neutrinos? Westview Press.

French, S. (2007). The limits of structuralism. BSPS Presidential Address: http://www. oxfordjournals. org/society/bsps/french_talk.

French, S. (2012). The presentation of objects and the representation of structure. In Structural realism, pages 3-28. Springer.

Gutiérrez-Ramírez, C. (2015). Is the Continum Hypothesis an absolutely undecidable proposition? A Philosophical Study? PhD thesis, UNAM, Mexico.

Hempel, C. G. (2000). Selected philosophical essays. Cambridge University Press.

Lakatos, I. (1978). The methodology of scientific research programmes. In Philosophical Papers. Cambridge University Press.

Laudan, L. (1977). Progress and its Problems. Ewing, NJ: University of California Press. 
Le Morvan, P. and Peels, R. (2016). The nature of ignorance: Two views. In The epistemic dimensions of ignorance. Cambridge: Cambridge University Press.

Martínez-Ordaz, M. (2017). Holism, inconsistency toleration and inconsistencies between theory and observation. HUMANA. MENTE Journal of Philosophical Studies, 10(32):117-147.

Meheus, J. (2002). How to reason sensibly yet naturally from inconsistencies. In Inconsistency in Science, pages 151-164. Springer.

Nottelmann, N. (2016). The varieties of ignorance. The epistemic dimensions of ignorance, pages $33-56$.

Peliš, M. (2017). Erotetic epistemic logic. Logic and Logical Philosophy, $26(3): 357-381$.

Pinch, T. (1986). Confronting nature: The sociology of solar-neutrino detection, volume 5. Springer Science \& Business Media.

Priest, G. (1998). What is so bad about contradictions? The Journal of Philosophy, 95(8):410-426.

Priest, G. (2002). Inconsistency and the empirical sciences. In Inconsistency in science, pages 119-128. Springer.

Pritchard, D. (2017). Epistemically useful false beliefs. Philosophical Explorations, 20(sup1):4-20.

Reck, E. H. and Price, M. P. (2000). Structures and structuralism in contemporary philosophy of mathematics. Synthese, 125(3):341-383.

Rescher, N. (2009). Ignorance:(On the wider implications of deficient knowledge). University of Pittsburgh Press. 